\title{
Data Dependence of Some New Iterative Schemes for Quasi-Contractive Operators
}

\author{
Renu Chugh, Sanjay Kumar and Jagjeet \\ Department of Mathematics \\ Maharshi Dayanand University \\ ROHTAK, INDIA
}

\begin{abstract}
In this paper we prove data dependence of new multistep iterative scheme as well as CR iterative scheme for quasi contractive operators, that is, by using an approximate quasi contractive operator we approximate the fixed point of the given operators.
\end{abstract}

\section{KEYWORDS:}

CR Iteration, Data Dependence, New Multistep Iteration, Quasi Contractive.

\section{Mathematics Subject Classification:} $47 \mathrm{H} 10$.

\section{INTRODUCTION}

Fixed point theory is involved in various types of issues such as to find the fixed point, existence and uniqueness of fixed point etc. Data dependence of fixed point is one of these issues and has become a subject of research interest from some time now. The data dependence of various iterative schemes has been studied by various authors like Rus and Muresan [8], Rus et al. [6, 7], Berinde[24], Espinola and Petrusel [17], Markin [10], Chifu and Petrusel [4], Olantiwo [11,12], Soltuz [19, 20], Soltuz and Grosan [21], Chugh and Kumar [15], Gursoy et al.[5] and several references thereof.

Our main interest in this paper is to show data dependence of new multi step [2] and CR [16] iterative schemes using quasi contractive operators. For the background of our exposition, we first mention some contractive mappings.

Zemfirescu [22] established a nice generalization of the Banach fixed point theorem as follows:

Let $(X, d)$ be a complete metric space and $\mathrm{T}: \mathrm{X} \rightarrow \mathrm{X}$ mappings for which there exists real numbers $\alpha, \beta, \gamma$ satisfying

$0 \leq \alpha<1,0 \leq \beta<\frac{1}{2}, 0 \leq \gamma<\frac{1}{2}$, respectively such that for each

$x, y \in E$, at least one of the following is true:

$$
\begin{aligned}
& \left(z_{1}\right) d(T x, T y) \leq \alpha d(x, y) \\
& \left(z_{2}\right) d(T x, T y) \leq \beta[d(x, T x)+d(y, T y)] \\
& \left(z_{3}\right) d(T x, T y) \leq \gamma[d(x, T y)+d(y, T x)]
\end{aligned}
$$

Then the mapping T satisfying (1.1) is called Zamfirescu contraction.

Remark 1.1. Mapping which satisfy $\left(z_{2}\right)$ is called a Kannan mapping, while the mapping satisfying $\left(\mathrm{z}_{3}\right)$ is called Chatterjea operator.
The contractive condition (1.1) implies

$$
\left\{\begin{array}{l}
\|T x-T y\| \leq 2 \delta\|x-T x\|+\delta\|x-y\| \\
\text { and } \\
\|T x-T y\| \leq 2 \delta\|x-T y\|+\delta\|x-y\|
\end{array} \text {, for all } \forall x, y \in E,\right.
$$

where $\delta=\max \left\{\alpha, \frac{\beta}{1-\beta}, \frac{\gamma}{1-\gamma}\right\}, 0 \leq \delta<1$.

Osilike and Udomene [14] defined a new general definition of quasi contractive operator as follows:

$$
\begin{aligned}
& \|T x-T y\| \leq \delta\|x-y\|+L\|x-T x\| \quad \forall x, y \in E \quad \text { and some } \\
& L \geq 0, \quad \delta \in[0,1] .
\end{aligned}
$$

After that a more general definition was introduced by Imoru and Olantiwo [3] as follows: if there exists a constant $0 \leq \delta<1$ and a monotonically increasing and continuous function $\varphi:[0, \infty) \rightarrow[0, \infty)$ with $\varphi(0)=0$, such that for $\forall x, y \in E$

$$
\|T x-T y\| \leq \delta\|x-y\|+\varphi(\|x-T x\|)
$$

Now we recapitulate some iterative schemes in the literature of fixed point theory. Let $\mathrm{X}$ be a Banach space and $\mathrm{E}$ be a closed, convex subset of $\mathrm{X}$. If $\mathrm{T}: \mathrm{X} \rightarrow \mathrm{X}$ a mappings on $\mathrm{E}$, then $F_{T}=\{p \in X: T p=p\}$ denotes the set of fixed points of $\mathrm{T}$.

For $x_{0} \in E$, Ishikawa Iteration [18], $\left\{x_{n}\right\}_{n=0}^{\infty}$ is defined as

$$
\begin{aligned}
& x_{n+1}=\left(1-\alpha_{n}\right) x_{n}+\alpha_{n} T y_{n} \\
& y_{n}=\left(1-\beta_{n}\right) x_{n}+\beta_{n} T x_{n},
\end{aligned}
$$

(1.5) where $\left\{\alpha_{n}\right\}_{n=0}^{\infty}$ and $\left\{\beta_{n}\right\}_{n=0}^{\infty}$ are real sequences in $[0,1]$ satisfying $\sum_{n=0}^{\infty} \alpha_{n}=\infty$.

Observe that if $\beta_{n}=0$ for each $n$, then the Ishikawa iteration process (1.5) reduces to the Mann iteration scheme. For $x_{0} \in E$, the Noor three step iterative scheme [13], $\left\{x_{n}\right\}_{n=0}^{\infty}$ is defined as

$$
\begin{aligned}
& x_{n+1}=\left(1-\alpha_{n}\right) x_{n}+T y_{n} \\
& y_{n}=\left(1-\beta_{n}\right) x_{n}+T z_{n} \\
& z_{n}=\left(1-\gamma_{n}\right) x_{n}+T x_{n},
\end{aligned}
$$


(1.6) where $\left\{\alpha_{n}\right\}_{n=0}^{\infty},\left\{\beta_{n}\right\}_{n=0}^{\infty}$ and $\left\{\gamma_{n}\right\}_{n=0}^{\infty}$ are real sequences in $[0,1]$ satisfying $\sum_{n=0}^{\infty} \alpha_{n}=\infty$.

If $\gamma_{\mathrm{n}}=0$, then Noor iteration process (1.6) reduces to Ishikawa Iteration scheme (1.5).

In 2007, Agarwal et al. defined the Agarwal et al. iterative scheme [1] as

$x_{n+1}=\left(1-\alpha_{n}\right) T x_{n}+\alpha_{n} T y_{n}$

$y_{n}=\left(1-\beta_{n}\right) x_{n}+\beta_{n} T x_{n}$,

where $\left\{\alpha_{n}\right\}$ and $\left\{\beta_{n}\right\}$ are sequences of positive numbers in

$[0,1]$ with $\sum_{n=0}^{\infty} \alpha_{n}=\infty$

Quite recently, Phuengrattana and Suantai [25] introduced SP iterative scheme as

$x_{n+1}=\left(1-\alpha_{n}\right) y_{n}+\alpha_{n} T y_{n}$

$y_{n}=\left(1-\beta_{n}\right) z_{n}+\beta_{n} T z_{n}$

$z_{n}=\left(1-\gamma_{n}\right) x_{n}+\gamma_{n} T x_{n}$

where $\left\{\alpha_{n}\right\},\left\{\beta_{n}\right\}$ and $\left\{\gamma_{n}\right\}$ are sequences of positive

numbers in $[0,1]$ satisfying $\sum_{n=0}^{\infty} \alpha_{n}=\infty$.

We shall use the following iterative schemes:

(a) New multi step iterative scheme

$\left\{\begin{array}{l}x_{n+1}=\left(1-\alpha_{n}\right) x_{n}+\alpha_{n} T_{1} y_{n}^{1} \\ y_{n}^{i}=\left(1-\beta_{n}^{i}\right) x_{n}+\beta_{n}^{i} T_{1} y_{n}^{i+1}, i=1,2 \ldots k-2 \\ y_{n}^{p-1}=\left(1-\beta_{n}^{k-1}\right) x_{n}+\beta_{n}^{k-1} T_{1} x_{n}\end{array}\right.$

and

$\left\{\begin{array}{l}u_{n+1}=\left(1-\alpha_{n}\right) u_{n}+\alpha_{n} T_{2} v_{n}^{1} \\ v_{n}^{i}=\left(1-\beta_{n}^{i}\right) u_{n}+\beta_{n}^{i} T_{2} v_{n}^{i+1}, i=1,2 \ldots . k-2 \\ v_{n}^{p-1}=\left(1-\beta_{n}^{k-1}\right) u_{n}+\beta_{n}^{k-1} T_{2} u_{n},\end{array}\right.$

where $\left\{\alpha_{n}\right\}_{n=0}^{\infty},\left\{\beta_{n}^{i}\right\}_{n=0}^{\infty}, i=1, . . k-2, k \geq 2$ be the real

sequences of positive numbers in $[0,1]$ satisfying $\sum_{n=0}^{\infty} \alpha_{n}=\infty$, due to Rhoades and Soltuz[2].

\section{(b) CR iterative scheme}

$$
\left\{\begin{array}{l}
x_{0} \in E \\
x_{n+1}=\left(1-\alpha_{n}\right) y_{n}+\alpha_{n} T_{1} y_{n} \\
y_{n}=\left(1-\beta_{n}\right) T_{1} x_{n}+\beta_{n} T_{1} z_{n} \\
z_{n}=\left(1-\gamma_{n}\right) x_{n}+\gamma_{n} T_{1} x_{n}
\end{array}\right.
$$$$
\text { and }
$$

$\left\{\begin{array}{l}u_{n+1}=\left(1-\alpha_{n}\right) v_{n}+\alpha_{n} T_{2} v_{n} \\ v_{n}=\left(1-\beta_{n}\right) T_{2} u_{n}+\beta_{n} T_{2} w_{n} \\ w_{n}=\left(1-\gamma_{n}\right) u_{n}+\gamma_{n} T_{2} u_{n}\end{array}\right.$ where $\left\{\alpha_{n}\right\},\left\{\beta_{n}\right\}$ and $\left\{\gamma_{n}\right\}$ are sequences of positive

numbers in $[0,1]$ satisfying $\sum_{n=0}^{\infty} \alpha_{n}=\infty$, due to Chugh and

Kumar [16].

Remark 1.2. By putting $\mathrm{k}=2$ and 3 , (1.9) reduces to (1.5) and (1.6) respectively.

Now to prove our main results we need following results in sequel.

Definition 1.3. [23] Let $T_{1}, T_{2}$ be two operators. We say $T_{2}$ is approximate operator of $\mathrm{T}_{1}$ if for all $x \in X$ and for a fixed

$\varepsilon>0$, we have $\left\|T_{1} x-T_{2} x\right\| \leq \varepsilon$.

Lemma 1.4.[21] Let $\left\{\alpha_{n}\right\}_{n=0}^{\infty}$ be a nonnegative sequence for which one suppose there exists $n_{0} \in I$, such that for all $n \geq n_{0}$ it satisfies the following inequality:

$\alpha_{n} \leq\left(1-\lambda_{n}\right) \alpha_{n}+\lambda_{n} \sigma_{n}$,

where $\lambda_{n} \in(0,1), \forall n \in N, \sum_{n=1}^{\infty} \lambda_{n}=\infty$ and $\sigma_{n} \geq 0 \forall n \in N$.

Then, $0 \leq \lim _{n \rightarrow \infty} \sup \alpha_{n} \leq \lim _{n \rightarrow \infty} \sup \sigma_{n}$.

Theorem 1.5. [16]: Let $\mathrm{T}: \mathrm{E} \rightarrow \mathrm{E}$ be a mapping satisfying (1.4) and $\left\{x_{n}\right\}_{n=0}^{\infty}$ be defined by (1.10) with real sequence, $\left\{\alpha_{n}\right\}_{n=0}^{\infty},\left\{\beta_{n}\right\}_{n=0}^{\infty}$ and $\left\{\gamma_{n}\right\}_{n=0}^{\infty}$ in $[0,1)$ satisfying $\sum_{n=0}^{\infty} \alpha_{n}=\infty$ .Then the sequence $\left\{x_{n}\right\}_{n=0}^{\infty}$ converges to unique fixed point of $\mathrm{T}$.

Theorem 1.6.[9]: Let $\mathrm{T}: \mathrm{E} \rightarrow \mathrm{E}$ be a mapping satisfying (1.4) and $\left\{x_{n}\right\}_{n=0}^{\infty}$ be defined by (1.9) with real sequence, $\left\{\alpha_{n}\right\}_{n=0}^{\infty}$,

$\left\{\beta_{n}^{i}\right\}_{n=0}^{\infty}, i=1, . . k-1$ in $[0,1)$ satisfying $\sum_{n=0}^{\infty} \alpha_{n}=\infty$.Then the sequence $\left\{x_{n}\right\}_{n=0}^{\infty}$ converges to unique fixed point of T.

\section{MAIN RESULTS}

Theorem 2.1. Let $T_{1}: E \rightarrow E$ be a mapping satisfying (1.4). Let $T_{2}$ be a approximate operator of $T_{1}$ as in Definition 1 and $\left\{x_{n}\right\}_{n=0}^{\infty},\left\{u_{n}\right\}_{n=0}^{\infty}$ be two CR iterative schemes defined by (1.10) associated to $T_{1}$ and $T_{2}$, respectively, where, $\left\{\alpha_{n}\right\}_{n=0}^{\infty}$, $\left\{\beta_{n}\right\}_{n=0}^{\infty}$ and $\left\{\gamma_{n}\right\}_{n=0}^{\infty}$ are real sequences in $[0,1)$ satisfying $\left\{\begin{array}{l}(i) \frac{1}{2} \leq \alpha_{n}(1-\delta), \forall n \\ \text { (ii) } \sum_{n=0}^{\infty} \alpha_{n}=\infty\end{array}\right.$. Let $p=T_{1} p$ and $q=T_{2} q$ then we have the following estimate:

$\|p-q\| \leq \frac{4 \varepsilon}{1-\delta}$.

Proof: Using (1.4) and (1.10), we have the following estimates: 


$$
\begin{aligned}
\left\|x_{n+1}-u_{n+1}\right\| & =\left\|\left(1-\alpha_{n}\right)\left(y_{n}-v_{n}\right)+\alpha_{n}\left(T_{1} y_{n}-T_{2} v_{n}\right)\right\| \\
& \leq\left(1-\alpha_{n}\right)\left\|y_{n}-v_{n}\right\|+\alpha_{n}\left\|T_{1} y_{n}-T_{2} v_{n}\right\| \\
& =\left(1-\alpha_{n}\right)\left\|y_{n}-v_{n}\right\|+\alpha_{n}\left\|T_{1} y_{n}-T_{1} v_{n}+T_{1} v_{n}-T_{2} v_{n}\right\| \\
& \leq\left(1-\alpha_{n}\right)\left\|y_{n}-v_{n}\right\|+\alpha_{n}\left\{\left\|T_{1} y_{n}-T_{1} v_{n}\right\|+\left\|T_{1} v_{n}-T_{2} v_{n}\right\|\right\} \\
& \leq\left(1-\alpha_{n}\right)\left\|y_{n}-v_{n}\right\|+\alpha_{n}\left\{\delta\left\|y_{n}-v_{n}\right\|+\varphi\left(\left\|T_{1} y_{n}-y_{n}\right\|\right)+\varepsilon\right\} \\
& \leq\left(1-\alpha_{n}(1-\delta)\right)\left\|y_{n}-v_{n}\right\|+\alpha_{n} \varphi\left(\left\|T_{1} y_{n}-y_{n}\right\|\right)+\alpha_{n} \varepsilon,
\end{aligned}
$$$$
\left\|y_{n}-v_{n}\right\|=\left\|\left(1-\beta_{n}\right)\left(T_{1} x_{n}-T_{2} u_{n}\right)+\beta_{n}\left(T_{1} z_{n}-T_{2} w_{n}\right)\right\|
$$$$
\leq\left(1-\beta_{n}\right)\left\|T_{1} x_{n}-T_{2} u_{n}\right\|+\beta_{n}\left\|T_{1} z_{n}-T_{2} w_{n}\right\|
$$$$
\leq\left(1-\beta_{n}\right)\left\|T_{1} x_{n}-T_{1} u_{n}+T_{1} u_{n}-T_{2} u_{n}\right\|
$$$$
+\beta_{n}\left\|T_{1} z_{n}-T_{1} w_{n}+T_{1} w_{n}-T_{2} w_{n}\right\|
$$$$
\leq\left(1-\beta_{n}\right)\left\{\left\|T_{1} x_{n}-T_{1} u_{n}\right\|+\left\|T_{1} u_{n}-T_{2} u_{n}\right\|\right\}
$$$$
+\beta_{n}\left\{\left\|T_{1} z_{n}-T_{1} w_{n}\right\|+\left\|T_{1} w_{n}-T_{2} w_{n}\right\|\right\}
$$$$
\leq\left(1-\beta_{n}\right)\left\{\delta\left\|x_{n}-u_{n}\right\|+\varphi\left(\left\|T_{1} x_{n}-x_{n}\right\|\right)+\varepsilon\right\}
$$$$
+\beta_{n}\left\{\delta\left\|z_{n}-w_{n}\right\|+\varphi\left(\left\|T_{1} z_{n}-z_{n}\right\|\right)+\varepsilon\right\}
$$$$
=\left(1-\beta_{n}\right) \delta\left\|x_{n}-u_{n}\right\|+\left(1-\beta_{n}\right) \varphi\left(\left\|T_{1} x_{n}-x_{n}\right\|\right)
$$$$
+\left(1-\beta_{n}\right) \varepsilon+\beta_{n} \delta\left\|z_{n}-w_{n}\right\|+\beta_{n} \varphi\left(\left\|T_{1} z_{n}-z_{n}\right\|\right)+\beta_{n} \varepsilon
$$

and

$$
\begin{aligned}
\left\|z_{n}-w_{n}\right\| & =\left\|\left(1-\gamma_{n}\right)\left(x_{n}-u_{n}\right)+\gamma_{n}\left(T_{1} x_{n}-T_{2} u_{n}\right)\right\| \\
& \leq\left(1-\gamma_{n}\right)\left\|x_{n}-u_{n}\right\|+\gamma_{n}\left\|T_{1} x_{n}-T_{2} u_{n}\right\| \\
& \leq\left(1-\gamma_{n}\right)\left\|x_{n}-u_{n}\right\|+\gamma_{n}\left\{\delta\left\|x_{n}-u_{n}\right\|+\varphi\left(\left\|T_{1} x_{n}-x_{n}\right\|\right)+\varepsilon\right\} \\
& =\left(1-\gamma_{n}(1-\delta)\right)\left\|x_{n}-u_{n}\right\|+\gamma_{n} \varphi\left(\left\|T_{1} x_{n}-x_{n}\right\|\right)+\gamma_{n} \varepsilon
\end{aligned}
$$

Combining (2.1), (2.2) and (2.3), we have

$$
\begin{gathered}
\left\|x_{n+1}-u_{n+1}\right\| \leq\left(1-\alpha_{n}(1-\delta)\right)\left\{\left(1-\beta_{n}\right) \delta\left\|x_{n}-u_{n}\right\|\right. \\
\left.+\left(1-\beta_{n}\right) \varphi\left(\left\|T_{1} x_{n}-x_{n}\right\|\right)+\left(1-\beta_{n}\right) \varepsilon\right\} \\
+\left(1-\alpha_{n}(1-\delta)\right)\left\{\beta_{n} \delta\left\|z_{n}-w_{n}\right\|+\beta_{n} \varphi\left(\left\|T_{1} z_{n}-z_{n}\right\|\right)+\beta_{n} \varepsilon\right\} \\
+\alpha_{n} \varphi\left(\left\|T_{1} y_{n}-y_{n}\right\|\right)+\alpha_{n} \varepsilon \\
=\left(1-\alpha_{n}(1-\delta)\right)\left(1-\beta_{n}\right) \delta\left\|x_{n}-u_{n}\right\| \\
+\left(1-\alpha_{n}(1-\delta)\right) \beta_{n} \delta\left\|z_{n}-w_{n}\right\| \\
+\left(1-\alpha_{n}(1-\delta)\right)\left(1-\beta_{n}\right) \varphi\left(\left\|T_{1} x_{n}-x_{n}\right\|\right) \\
+\left(1-\alpha_{n}(1-\delta)\right)\left(1-\beta_{n}\right) \varepsilon \\
+\left(1-\alpha_{n}(1-\delta)\right) \beta_{n} \varphi\left(\left\|T_{1} z_{n}-z_{n}\right\|\right) \\
+\left(1-\alpha_{n}(1-\delta)\right) \beta_{n} \varepsilon \\
+\alpha_{n} \varphi\left(\left\|T_{1} y_{n}-y_{n}\right\|\right)+\alpha_{n} \varepsilon
\end{gathered}
$$

$$
\begin{gathered}
=\left(1-\alpha_{n}(1-\delta)\right)\left(1-\beta_{n}\right) \delta\left\|x_{n}-u_{n}\right\| \\
+\left(1-\alpha_{n}(1-\delta)\right) \beta_{n} \delta\left\{\left(1-\gamma_{n}(1-\delta)\right)\left\|x_{n}-u_{n}\right\|\right. \\
\left.+\gamma_{n} \varphi\left(\left\|T_{1} x_{n}-x_{n}\right\|\right)+\gamma_{n} \varepsilon\right\} \\
+\left(1-\alpha_{n}(1-\delta)\right)\left(1-\beta_{n}\right) \varphi\left(\left\|T_{1} x_{n}-x_{n}\right\|\right) \\
+\left(1-\alpha_{n}(1-\delta)\right)\left(1-\beta_{n}\right) \varepsilon \\
+\left(1-\alpha_{n}(1-\delta)\right) \beta_{n} \varphi\left(\left\|T_{1} z_{n}-z_{n}\right\|\right) \\
+\left(1-\alpha_{n}(1-\delta)\right) \beta_{n} \varepsilon+\alpha_{n} \varphi\left(\left\|T_{1} y_{n}-y_{n}\right\|\right)+\alpha_{n} \varepsilon \\
+\left\{\left(1-\alpha_{n}(1-\delta)\right)\left(1-\beta_{n}(1-\delta)\left(1-\gamma_{n}(1-\delta)\right)\right)\right\}\left\|x_{n}-u_{n}\right\| \\
+\delta\left(1-\alpha_{n}(1-\delta)\right) \beta_{n} \gamma_{n} \varphi\left(\left\|T_{1} x_{n}-x_{n}\right\|\right) \\
+\left(1-\alpha_{n}(1-\delta)\right) \beta_{n} \delta \gamma_{n} \varepsilon \\
+\left(1-\alpha_{n}(1-\delta)\right)\left(1-\beta_{n}\right) \varphi\left(\left\|T_{1} x_{n}-x_{n}\right\|\right) \\
+\left(1-\alpha_{n}(1-\delta)\right)\left(1-\beta_{n}\right) \varepsilon \\
+\left(1-\alpha_{n}(1-\delta)\right) \beta_{n} \varphi\left(\left\|T_{1} z_{n}-z_{n}\right\|\right) \\
+\left(1-\alpha_{n}(1-\delta)\right) \beta_{n} \varepsilon+\alpha_{n} \varphi\left(\left\|T_{1} y_{n}-y_{n}\right\|\right) \\
+\alpha_{n} \varepsilon
\end{gathered}
$$

It may be noted that for $\left\{\alpha_{n}\right\}_{n=0}^{\infty},\left\{\beta_{n}\right\}_{n=0}^{\infty}$,

$\left\{\gamma_{n}\right\}_{n=0}^{\infty} \subset[0,1)$ and $0 \leq \delta<1$, the following inequalities hold:

$\left\{\begin{array}{l}\left(1-\alpha_{n}\right) \delta<\left(1-\alpha_{n}\right), \\ \left(1-\beta_{n}(1-\delta)\left(1-\gamma_{n}(1-\delta)\right)\right)<1, \\ \alpha_{n} \beta_{n} \delta<\alpha_{n} .\end{array}\right.$

It follows from assumption (i) that

$\left(1-\alpha_{n}(1-\delta)\right)<\alpha_{n}(1-\delta) \leq \alpha_{n}, \forall n \in I$

Now using (2.5) and (2.6) in (2.4), we get

$$
\begin{aligned}
\left\|x_{n+1}-u_{n+1}\right\| \leq & \left(1-\alpha_{n}(1-\delta)\right)\left\|x_{n}-u_{n}\right\|+2 \alpha_{n} \varphi\left(\left\|T_{1} x_{n}-x_{n}\right\|\right) \\
& +4 \alpha_{n} \varepsilon+2 \alpha_{n} \varphi\left(\left\|T_{1} z_{n}-z_{n}\right\|\right)+\alpha_{n} \varphi\left(\left\|T_{1} y_{n}-y_{n}\right\|\right)
\end{aligned}
$$

which further implies

$$
\begin{aligned}
& \left\|x_{n+1}-u_{n+1}\right\| \leq\left(1-\alpha_{n}(1-\delta)\right)\left\|x_{n}-u_{n}\right\| \\
& \quad+\alpha_{n}(1-\delta) \frac{\left\{2 \varphi\left(\left\|T_{1} x_{n}-x_{n}\right\|\right)+4 \varepsilon+\varphi\left(\left\|T_{1} z_{n}-z_{n}\right\|\right)+\varphi\left(\left\|T_{1} y_{n}-y_{n}\right\|\right)\right\}}{(1-\delta)}
\end{aligned}
$$


Let us denote

$a_{n}=\left\|x_{n}-u_{n}\right\|$

$r_{n}=\alpha_{n}(1-\delta)$

and

$\sigma_{n}=\frac{\left\{2 \varphi\left(\left\|T_{1} x_{n}-x_{n}\right\|\right)+4 \varepsilon+\varphi\left(\left\|T_{1} z_{n}-z_{n}\right\|\right)+\varphi\left(\left\|T_{1} y_{n}-y_{n}\right\|\right)\right\}}{(1-\delta)}$.

Now from Theorem 1 we have $\lim _{n \rightarrow \infty}\left\|x_{n}-p\right\|=0$. Also $\mathrm{T}$

satisfies condition (1.4) and $T p=p \in F_{T}$, hence

$\lim _{n \rightarrow \infty}\left\|x_{n}-T x_{n}\right\|=\lim _{n \rightarrow \infty}\left\|y_{n}-T y_{n}\right\|=\lim _{n \rightarrow \infty}\left\|z_{n}-T z_{n}\right\|=0$.

Because $\left\{x_{n}\right\}_{n=0}^{\infty},\left\{y_{n}\right\}_{n=0}^{\infty},\left\{z_{n}\right\}_{n=0}^{\infty}$ converges to fixed point of $\mathrm{T}$

$\lim _{n \rightarrow \infty} \varphi\left(\left\|x_{n}-T x_{n}\right\|\right)=\lim _{n \rightarrow \infty} \varphi\left(\left\|y_{n}-T y_{n}\right\|\right)=\lim _{n \rightarrow \infty} \varphi\left(\left\|z_{n}-T z_{n}\right\|\right)=0$.

Since $\varphi$ is continuous, hence using Lemma 1, (2.7) yields

$\|p-q\| \leq \frac{4 \varepsilon}{(1-\delta)}$.

Theorem 2.2. Let $T_{1}: E \rightarrow E$ be a mapping satisfying (1.4).

Let $T_{2}$ be a approximate operator of $T_{1}$ as in Definition 1 and

$\left\{x_{n}\right\}_{n=0}^{\infty},\left\{u_{n}\right\}_{n=0}^{\infty}$ be two iterative schemes defined by (1.9) associated to $T_{1}$ and $T_{2}$ respectively, where, $\left\{\alpha_{n}\right\}_{n=0}^{\infty}$ and $\left\{\beta_{n}^{i}\right\}_{n=0}^{\infty}, i=1, \ldots k-1$ are real sequences in $[0,1)$ satisfying

$\int(i) o \leq \beta_{n}^{i} \leq \alpha_{n}<1, i=1, . . k-1$

$\left\{\right.$ (ii) $\sum_{n=0}^{\infty} \alpha_{n}=\infty \quad$. Let $p=T_{1} p$ and $q=T_{2} q$

then we have the following estimate

$\|p-q\| \leq \frac{(k-1) \varepsilon}{1-\delta}$.

Proof: For a given $x_{0} \in E$ and $u_{0} \in E$ we consider the following iterative schemes for $T_{1}$ and $T_{2}$

$$
\begin{aligned}
& \left\{\begin{array}{l}
x_{n+1}=\left(1-\alpha_{n}\right) x_{n}+\alpha_{n} T_{1} y_{n}^{1} \\
y_{n}^{i}=\left(1-\beta_{n}^{i}\right) x_{n}+\beta_{n}^{i} T_{1} y_{n}^{i+1}, i=1,2 \ldots . k-2 \\
y_{n}^{p-1}=\left(1-\beta_{n}^{p-1}\right) x_{n}+\beta_{n}^{p-1} T_{1} x_{n},
\end{array}\right. \\
& \left\{\begin{array}{l}
u_{n+1}=\left(1-\alpha_{n}\right) u_{n}+\alpha_{n} T_{2} v_{n}^{1} \\
v_{n}^{i}=\left(1-\beta_{n}^{i}\right) u_{n}+\beta_{n}^{i} T_{2} v_{n}^{i+1}, i=1,2 \ldots . k-2 \\
v_{n}^{p-1}=\left(1-\beta_{n}^{p-1}\right) u_{n}+\beta_{n}^{p-1} T_{2} u_{n},
\end{array}\right.
\end{aligned}
$$

then using (1.4), (2.8) and (2.9), yield the following estimates:

$$
\begin{aligned}
\left\|x_{n+1}-u_{n+1}\right\| & =\left\|\left(1-\alpha_{n}\right)\left(x_{n}-u_{n}\right)+\alpha_{n}\left(T_{1} y_{n}^{1}-T_{2} v_{n}^{1}\right)\right\| \\
& \leq\left(1-\alpha_{n}\right)\left\|x_{n}-u_{n}\right\|+\alpha_{n}\left\|T_{1} y_{n}^{1}-T_{2} v_{n}^{1}\right\| \\
& =\left(1-\alpha_{n}\right)\left\|x_{n}-u_{n}\right\|+\alpha_{n}\left\|T_{1} y_{n}^{1}-T_{1} v_{n}^{1}+T_{1} v_{n}^{1}-T_{2} v_{n}^{1}\right\| \\
& \leq\left(1-\alpha_{n}\right)\left\|x_{n}-u_{n}\right\|+\alpha_{n}\left\|T_{1} y_{n}^{1}-T_{1} v_{n}^{1}\right\|+\alpha_{n}\left\|T_{1} v_{n}^{1}-T_{2} v_{n}^{1}\right\| \\
& \leq\left(1-\alpha_{n}\right)\left\|x_{n}-u_{n}\right\|+\alpha_{n} \delta\left\|y_{n}^{1}-v_{n}^{1}\right\|+\alpha_{n} \varphi\left(\left\|y_{n}^{1}-T_{1} y_{n}^{1}\right\|\right)+\alpha_{n} \varepsilon
\end{aligned}
$$

$$
\begin{aligned}
\left\|y_{n}^{1}-v_{n}^{1}\right\| & =\left\|\left(1-\beta_{n}^{1}\right)\left(x_{n}-u_{n}\right)+\beta_{n}^{1}\left(T_{1} y_{n}^{2}-T_{2} v_{n}^{2}\right)\right\| \\
& \leq\left(1-\beta_{n}^{1}\right)\left\|x_{n}-u_{n}\right\|+\beta_{n}^{1}\left\|T_{1} y_{n}^{2}-T_{2} v_{n}^{2}\right\| \\
& \leq\left(1-\beta_{n}^{1}\right)\left\|x_{n}-u_{n}\right\|+\beta_{n}^{1} \delta\left\|T_{1} y_{n}^{2}-T_{2} v_{n}^{2}\right\|+\beta_{n}^{1} \varphi\left(\left\|y_{n}^{2}-T_{1} y_{n}^{2}\right\|\right)+\beta_{n}^{1} \varepsilon
\end{aligned}
$$

and

$$
\begin{aligned}
\left\|y_{n}^{2}-v_{n}^{2}\right\| & =\left\|\left(1-\beta_{n}^{2}\right)\left(x_{n}-u_{n}\right)+\beta_{n}^{2}\left(T_{1} y_{n}^{3}-T_{2} v_{n}^{3}\right)\right\| \\
& \leq\left(1-\beta_{n}^{2}\right)\left\|x_{n}-u_{n}\right\|+\beta_{n}^{2}\left\|T_{1} y_{n}^{3}-T_{2} v_{n}^{3}\right\| \\
& \leq\left(1-\beta_{n}^{2}\right)\left\|x_{n}-u_{n}\right\|+\beta_{n}^{2} \delta\left\|y_{n}^{3}-v_{n}^{3}\right\|+\beta_{n}^{2} \varphi\left(\left\|y_{n}^{3}-T_{1} y_{n}^{3}\right\|\right)+\beta_{n}^{2} \varepsilon
\end{aligned}
$$

Combining (2.10), (2.11) and (2.12), we have

$$
\begin{aligned}
\left\|x_{n+1}-u_{n+1}\right\| \leq & \left(1-\alpha_{n}\right)\left\|x_{n}-u_{n}\right\|+\alpha_{n} \varphi\left(\left\|y_{n}^{1}-T_{1} y_{n}^{1}\right\|\right)+\alpha_{n} \varepsilon \\
& +\alpha_{n} \delta\left\{\left(1-\beta_{n}^{1}\right)\left\|x_{n}-u_{n}\right\|+\beta_{n}^{1} \delta\left\|y_{n}^{2}-v_{n}^{2}\right\|\right. \\
& \left.+\beta_{n}^{1} \varphi\left(\left\|y_{n}^{2}-T_{1} y_{n}^{2}\right\|\right)+\beta_{n}^{1} \varepsilon\right\} \\
\leq & \left.\left(1-\alpha_{n}(1-\delta)\right)-\delta \alpha_{n} \beta_{n}^{1}\right]\left\|x_{n}-u_{n}\right\| \\
+ & \delta^{2} \alpha_{n} \beta_{n}^{1}\left\|y_{n}^{2}-v_{n}^{2}\right\|+\alpha_{n} \varphi\left(\left\|y_{n}^{1}-T_{1} y_{n}^{1}\right\|\right) \\
& +\delta \alpha_{n} \beta_{n}^{1} \varphi\left(\left\|y_{n}^{2}-T_{1} y_{n}^{2}\right\|\right)+\alpha_{n} \varepsilon+\delta \alpha_{n} \beta_{n}^{1} \varepsilon \\
\leq & \left.\left(1-\alpha_{n}(1-\delta)\right)-\delta \alpha_{n} \beta_{n}^{1}\left(1-\delta\left(1-\beta_{n}^{2}\right)\right)\right]\left\|x_{n}-u_{n}\right\| \\
& +\delta^{3} \alpha_{n} \beta_{n}^{1} \beta_{n}^{2}\left\|y_{n}^{3}-v_{n}^{3}\right\|+\alpha_{n} \varphi\left(\left\|y_{n}^{1}-T_{1} y_{n}^{1}\right\|\right) \\
& +\delta \alpha_{n} \beta_{n}^{1} \varphi\left(\left\|y_{n}^{2}-T_{1} y_{n}^{2}\right\|\right)+\delta^{3} \alpha_{n} \beta_{n}^{1} \beta_{n}^{2} \varphi\left(\left\|y_{n}^{3}-T_{1} y_{n}^{3}\right\|\right) \\
& +\alpha_{n} \varepsilon+\delta \alpha_{n} \beta_{n}^{1} \varepsilon+\delta \alpha_{n} \beta_{n}^{1} \beta_{n}^{2}
\end{aligned}
$$

Thus inductively, we get

$\left\|x_{n+1}-u_{n+1}\right\| \leq$

$$
\begin{aligned}
& {\left[\left(1-\alpha_{n}(1-\delta)\right)-\delta \alpha_{n} \beta_{n}^{1}\left(1-\delta\left(1-\beta_{n}^{2}\right)-\delta^{2} \beta_{n}^{2}\left(1-\beta_{n}^{3}\right)-\right.\right.} \\
& \left.\left.\ldots \ldots . .-\delta^{p-4} \beta_{n}^{2} \beta_{n}^{3} \ldots \beta_{n}^{k-4}\left(1-\beta_{n}^{k-3}\right)\right)\right]\left\|x_{n}-u_{n}\right\| \\
& +\delta^{k-3} \alpha_{n} \beta_{n}^{1} \beta_{n}^{2} \ldots \beta_{n}^{k-4}\left\|y_{n}^{k-2}-v_{n}^{k-2}\right\| \\
& +\alpha_{n} \varphi\left(\left\|y_{n}^{1}-T_{1} y_{n}^{1}\right\|\right)+\delta \alpha_{n} \beta_{n}^{1} \varphi\left(\left\|y_{n}^{2}-T_{1} y_{n}^{2}\right\|\right) \\
& +\delta^{3} \alpha_{n} \beta_{n}^{1} \beta_{n}^{2} \varphi\left(\left\|y_{n}^{3}-T_{1} y_{n}^{3}\right\|\right)+ \\
& \quad \ldots \ldots+\delta^{k-3} \alpha_{n} \beta_{n}^{1} \beta_{n}^{2} \ldots \beta_{n}^{k-3} \varphi\left(\left\|y_{n}^{k-2}-T_{1} y_{n}^{k-2}\right\|\right) \\
& +\alpha_{n} \varepsilon+\delta \alpha_{n} \beta_{n}^{1} \varepsilon+\delta \alpha_{n} \beta_{n}^{1} \beta_{n}^{2} \varepsilon+ \\
& \quad \ldots .+\delta^{k-3} \alpha_{n} \beta_{n}^{1} \beta_{n}^{2} \ldots \beta_{n}^{k-3} \varepsilon
\end{aligned}
$$

Using (1.4) and (1.9)

$$
\begin{aligned}
\left\|y_{n}^{k-2}-v_{n}^{k-2}\right\| & \leq\left\|\left(1-\beta_{n}^{k-2}\right)\left(x_{n}-u_{n}\right)+\beta_{n}^{k-2}\left(T_{1} x_{n}-T_{2} u_{n}\right)\right\| \\
& \leq\left(1-\beta_{n}^{k-2}\right)\left\|x_{n}-u_{n}\right\|+\beta_{n}^{k-2}\left\|T_{1} x_{n}-T_{2} u_{n}\right\| \\
& \leq\left(1-\beta_{n}^{k-2}(1-\delta)\right)\left\|x_{n}-u_{n}\right\|+\beta_{n}^{k-2} \varphi\left(\left\|x_{n}-T_{1} x_{n}\right\|\right)+\beta_{n}^{k-2} \varepsilon
\end{aligned}
$$

Now by combining (2.14) and (2.15)

$\left\|x_{n+1}-u_{n+1}\right\| \leq$

$$
\begin{aligned}
& {\left[\left(1-\alpha_{n}(1-\delta)\right)-\delta \alpha_{n} \beta_{n}^{1}\left(1-\delta\left(1-\beta_{n}^{2}\right)-\right.\right.} \\
& \left.\quad \ldots .-\delta^{k-4} \beta_{n}^{2} \ldots \beta_{n}^{k-3}\left(1-\beta_{n}^{k-2}(1-\delta)\right)\right]\left\|x_{n}-u_{n}\right\| \\
& +\alpha_{n} \varphi\left(\left\|y_{n}^{1}-T_{1} y_{n}^{1}\right\|\right)+\delta \alpha_{n} \beta_{n}^{1} \varphi\left(\left\|y_{n}^{2}-T_{1} y_{n}^{2}\right\|\right) \\
& \quad+\delta^{3} \alpha_{n} \beta_{n}^{1} \beta_{n}^{2} \varphi\left(\left\|y_{n}^{3}-T_{1} y_{n}^{3}\right\|\right)+ \\
& \quad \ldots . .+\delta^{k-2} \alpha_{n} \beta_{n}^{1} \ldots \beta_{n}^{k-2} \varphi\left(\left\|x_{n}-T_{1} x_{n}\right\|\right) \\
& \quad+\alpha_{n} \varepsilon+\delta \alpha_{n} \beta_{n}^{1} \varepsilon+\ldots .+\delta^{k-2} \alpha_{n} \beta_{n}^{1} \ldots \beta_{n}^{k-2} \varepsilon,
\end{aligned}
$$


which further implies

$$
\begin{aligned}
\left\|x_{n+1}-u_{n+1}\right\| \leq[ & \left.\left(1-\alpha_{n}(1-\delta)\right)-\delta \alpha_{n} \beta_{n}^{1}+L\right]\left\|x_{n}-u_{n}\right\| \\
+ & \alpha_{n} \varphi\left(\left\|y_{n}^{1}-T_{1} y_{n}^{1}\right\|\right)+\delta \alpha_{n} \beta_{n}^{1} \varphi\left(\left\|y_{n}^{2}-T_{1} y_{n}^{2}\right\|\right) \\
+ & \delta^{3} \alpha_{n} \beta_{n}^{1} \beta_{n}^{2} \varphi\left(\left\|y_{n}^{3}-T_{1} y_{n}^{3}\right\|\right)+\ldots \ldots \\
& +\delta^{k-2} \alpha_{n} \beta_{n}^{1} \ldots \beta_{n}^{k-2} \varphi\left(\left\|x_{n}-T_{1} x_{n}\right\|\right) \\
& +\alpha_{n} \varepsilon+\delta \alpha_{n} \beta_{n}^{1} \varepsilon+\ldots .+\delta^{p-2} \alpha_{n} \beta_{n}^{1} \ldots \beta_{n}^{k-2} \varepsilon,
\end{aligned}
$$

where

$$
\left\{\begin{aligned}
L & =\delta^{2} \alpha_{n} \beta_{n}^{1}\left(1-\beta_{n}^{2}\right) \ldots .+\delta^{p-3} \alpha_{n} \beta_{n}^{1} \beta_{n}^{2} \ldots \beta_{n}^{p-3}\left(1-\beta_{n}^{k-2}(1-\delta)\right) \\
& \leq \delta^{2} \alpha_{n} \beta_{n}^{1} \beta_{n}^{2} \ldots .+\delta^{k-3} \alpha_{n} \beta_{n}^{1} \beta_{n}^{2} \ldots \beta_{n}^{k-3} \\
& \leq \delta^{2} \alpha_{n} \beta_{n}^{1} \beta_{n}^{2}+\delta^{3} \alpha_{n} \beta_{n}^{1} \beta_{n}^{2} \beta_{n}^{3} \ldots .+\delta^{k-3} \alpha_{n} \beta_{n}^{1} \beta_{n}^{2} \ldots \beta_{n}^{k-3} \\
& \leq \delta^{2} \alpha_{n} \beta_{n}^{1} \beta_{n}^{2}+\delta^{3} \alpha_{n} \beta_{n}^{1} \beta_{n}^{2} \ldots+\delta^{k-3} \alpha_{n} \beta_{n}^{1} \beta_{n}^{2} \\
& =\delta^{2} \alpha_{n} \beta_{n}^{1} \beta_{n}^{2}\left[1+\delta+\delta^{2}+\ldots \delta^{k-5}\right] \\
& =\delta^{2} \alpha_{n} \beta_{n}^{1} \beta_{n}^{2} \frac{\left[1-\delta^{k-4}\right]}{[1-\delta]}<\delta \alpha_{n} \beta_{n}^{1}
\end{aligned}\right.
$$

Since $\delta \frac{\left[1-\delta^{k-3}\right]}{[1-\delta]}<1$, this imply

$$
\left\{\left(1-\alpha_{n}(1-\delta)\right)-\delta \alpha_{n} \beta_{n}^{1}+L<\left(1-\alpha_{n}(1-\delta)\right) .\right.
$$

Hence using (2.17) and (2.18) in (2.16), we get $\left\|x_{n+1}-u_{n+1}\right\| \leq\left[1-\left(1-\alpha_{n}(1-\delta)\right)\right]\left\|x_{n}-u_{n}\right\|$

$$
\begin{gathered}
+\alpha_{n} \varphi\left(\left\|y_{n}^{1}-T_{1} y_{n}^{1}\right\|\right)+\alpha_{n} \varphi\left(\left\|y_{n}^{2}-T_{1} y_{n}^{2}\right\|\right) \\
+\alpha_{n} \varphi\left(\left\|y_{n}^{3}-T_{1} y_{n}^{3}\right\|\right)+\ldots \ldots \\
+\alpha_{n} \varphi\left(\left\|x_{n}-T_{1} x_{n}\right\|\right)+(k-1) \varepsilon \\
=\left[1-\left(1-\alpha_{n}(1-\delta)\right)\right]\left\|x_{n}-u_{n}\right\| \\
+\alpha_{n}(1-\delta)\left\{\frac{\varphi\left(\left\|y_{n}^{1}-T_{1} y_{n}^{1}\right\|\right)+\varphi\left(\left\|y_{n}^{2}-T_{1} y_{n}^{2}\right\|\right)+. .+\alpha_{n} \varphi\left(\left\|x_{n}-T_{1} x_{n}\right\|\right)+(k-1) \varepsilon}{(1-\delta)}\right\}
\end{gathered}
$$

Let us denote

$$
a_{n}=\left\|x_{n}-u_{n}\right\|
$$$$
r_{n}=\alpha_{n}(1-\delta) \text {, }
$$

and

$$
\sigma_{n}: \frac{\left\{\varphi\left(\left\|y_{n}^{1}-T_{1} y_{n}^{1}\right\|\right)+\varphi\left(\left\|y_{n}^{2}-T_{1} y_{n}^{2}\right\|\right)+\ldots \ldots+\varphi\left(\left\|x_{n}-T_{1} x_{n}\right\|\right)+(k-1) \varepsilon\right\}}{(1-\delta)}
$$

Now from Theorem 2, we have $\lim _{n \rightarrow \infty}\left\|x_{n}-p\right\|=0$. Also T satisfies condition (1.4) and $T p=p \in F_{T}$, using the similar argument as in Theorem 3, we get,

$\lim _{n \rightarrow \infty}\left\|x_{n}-T_{1} x_{n}\right\|=\lim _{n \rightarrow \infty}\left\|y_{n}^{1}-T_{1} y_{n}^{1}\right\|=\lim _{n \rightarrow \infty}\left\|y_{n}^{2}-T_{1} y_{n}^{2}\right\|=\ldots=\lim _{n \rightarrow \infty}\left\|y_{n}^{k-1}-T_{1} y_{n}^{k-1}\right\|=0$

Since $\varphi$ is continuous, we have

$$
\begin{gathered}
\lim _{n \rightarrow \infty} \varphi\left(\left\|x_{n}-T_{1} x_{n}\right\|\right)=\lim _{n \rightarrow \infty} \varphi\left(\left\|y_{n}^{1}-T_{1} y_{n}^{1}\right\|\right)=\lim _{n \rightarrow \infty} \varphi\left(\left\|y_{n}^{2}-T_{1} y_{n}^{2}\right\|\right) \\
=. .=\lim _{n \rightarrow \infty} \varphi\left(\left\|y_{n}^{k-1}-T_{1} y_{n}^{k-1}\right\|\right)=0
\end{gathered}
$$

Hence using Lemma 1, (2.19) yields

$\|p-q\| \leq \frac{(k-1) \varepsilon}{1-\delta}$

Remark 2.3. Since the iteration (1.5) and (1.6) are special cases of iterative scheme (1.9), so. Theorem 2 generalizes existing result for (1.5) and (1.6). By taking $\mathrm{k}=3$ and $\mathrm{k}=2$ in Theorem 4, data dependence results for the iterative schemes (1.5) and (1.6) can be obtained easily.

Numerical example 2.1.[21]. Let $\mathrm{T}: \mathrm{R} \rightarrow \mathrm{R}$ be a quasi contractive operator defined by

$$
T(x)=\left\{\begin{array}{ll}
0 & \text { if } x \in(-\infty, 2] \\
-0.5 & \text { if } x \in(2, \infty)
\end{array},\right.
$$

with $\mathrm{q}=0.2, \varphi=$ identity and having unique fixed point zero.

Now consider mapping $\mathbf{S}: \mathrm{R} \rightarrow \mathrm{R}$ defined by

$$
S(x)=\left\{\begin{array}{ll}
1 & \text { if } x \in(-\infty, 2] \\
-1.5 & \text { if } x \in(2, \infty)
\end{array},\right.
$$

with unique fixed point one. Let us assume $\varepsilon=1$, distance between two maps as follows:

$\|S x-T x\| \leq 1, \forall x \in R$.

if $u_{0}=x_{0}=0$ and $\alpha_{n}, \beta_{n}, \gamma_{n}=\frac{1}{n+1}$ then by using executable program in $\mathrm{C}++$ we get following table for $\mathrm{CR}$ and new multi step iterative schemes. 
Table 2.1

\begin{tabular}{|c|c|c|c|}
\hline $\begin{array}{c}\text { Number of } \\
\text { Iterative Step }\end{array}$ & $\begin{array}{c}\text { New Multi step } \\
\text { Iteration }\end{array}$ & $\begin{array}{c}\text { Number of } \\
\text { Iterative Step }\end{array}$ & CR Iteration \\
\hline 1 & 0.707107 & 1 & 1 \\
\hline 2 & 0.914214 & 2 & 1 \\
\hline 3 & 0.974874 & 3 & 1 \\
\hline 4 & 0.992641 & 4 & 1 \\
\hline 5 & 0.997845 & - & 1 \\
\hline 6 & 0.999369 & - & - \\
\hline 7 & 0.999815 & - & - \\
\hline 8 & 0.999946 & - & - \\
\hline 9 & 0.999984 & - & - \\
\hline 10 & 0.999995 & - & - \\
\hline 11 & 0.999999 & & \\
\hline 12 & 1 & & \\
\hline 13 & 1 & & \\
\hline
\end{tabular}

When new multi step and CR iterative schemes applied to $S$ it converges to unique fixed point $\mathrm{x}^{*}=1$.Also the distance between the

fixed point of $\mathrm{S}$ and $\mathrm{T}$ is one therefore if $\varepsilon=\frac{1}{4}$ then without

computing the fixed point of $S$, from theorem 3.1 we have the following estimates:

$\left\|x^{*}-u^{*}\right\| \leq \frac{1}{1-q}=\frac{1}{1-0.2}=1.2$.

Also, if $\varepsilon=\frac{1}{1-k}$, then from theorem 3.2 we have the

following estimates:

$\left\|x^{*}-u^{*}\right\| \leq \frac{1}{1-q}=\frac{1}{1-0.2}=1.2$.

\section{REFERENCES}

[1] Agarwal, R.P., O'Regan, D. and Sahu, D.R.: Iterative construction of fixed points of nearly asymptotically nonexpansive mappings, Journal of Nonlinear and Convex Analysis 8(1) (2007), 61-79.

[2] B.E. Rhoades, S.M. Soltuz, "The equivalence between Mann-Ishikawa iterations and multi-step iteration", Nonlinear Analysis, 58(2004), 219-228.

[3] C.O. Imoru, M.O. Olantiwo, "On the stability of Picard and Mann iteration processes", Carpathian Journal of Mathematics, 19(2003), no.2, 155-160.

[4] Chifu, G. Petru, sel, "Existence and Data Dependence of Fixed Points and Strict Fixed Points for ContractiveType Multivalued Operators", Fixed Point Theory and Applications, 2007(2007), Article ID 034248, 8 pages.

[5] F. Gursoy et al., "Data dependence results of new multistep and s-iterative schemes for contractive-like operators", Fixed Point Theory and Application, 10.1186/1687-1812-2013-76.

[6] I.A. Rus, A. Petru,sel, A. S^ıntamarian,"Data dependence of the fixed points set of multivalued weakly Picard operators", Stud. Univ. Babes-Bolyai, Math. 46 (2) (2001) 111-121.
[7] I.A. Rus, A. Petru, sel, A. S^1ntamarian, "Data dependence of the fixed point set of some multivalued weakly Picard operators", Nonlinear Analysis: Theory, Methods \& Applications, 52 (2003), 1947-1959

[8] I.A. Rus, S. Muresan,'Data dependence of the fixed points set of weakly Picard operators", Stud. Univ. BabesBolyai 43 (1998) 79-83.

[9] J.O. Olaleru, H. Akewe," On multistep iterative scheme for approximating the common fixed points of contractive-like operators", Int. Journal of Mathematics and mathematical sciences. Volume 2010, Article ID 530964, 11 pages

[10] J.T. Markin,"Continuous dependence of fixed point sets", Proc. AMS 38 (1973) 545-547.

[11] M. O. Olatinwo,"On the continuous dependence of the fixed points for $(\varphi, \psi)$-contractive-type operators", Kragujevac Journal of Mathematics, 34(2010), 91-102.

[12] M. O. Olatinwo,"Some results on the continuous dependence of the fixed points in normed linear space", Fixed Point Theory, 10(2009), no. 1, 151-157.

[13] M.A. Noor,"New approximation schemes for general variational inequalities", Journal of Mathematical Analysis and Applications, 251(2000), no.1, 217-229.

[14] M.O. Osilike, A. Udomene, "Short proofs of stability results for fixed point iteration procedures for a class of contractive-type mappings", Indian Journal of Pure and Applied Mathematics, 30(1999), no.12, 1229-1234.

[15] R. Chugh, V. Kumar, "Data dependence of Noor and SP iterative schemes when dealing with quasi-contractive operators", International Journal of Computer Applications, 31(2011), no.5.

[16] R. Chugh, V. Kumar, S. Kumar,"Strong convergence of a new three step iterative scheme in Banach spaces", American Journal of Computational Mathematics, 2(2012), 345-357.

[17] R. Esp'inola, A. Petru, sel,'Existence and data dependence of fixed points for multivalued operators on gauge spaces”, J. Math. Anal. Appl., 309 (2005), 420432 . 
[18] S. Ishikawa,"Fixed points by a new iteration method", Proc. Amer. Math. Soc., 44(1974), 147-150.

[19] S.M. Soltuz,'Data dependence for Ishikawa iteration", Lecturas Mathematicas, 25(2004), no. 2, 149-155.

[20] S.M. Soltuz, "Data dependence for Mann iteration", Octogon Math. Magazine, 9(2001), 825-828.

[21] S.M. Soltuz, T. Grosan,'Data dependence for Ishikawa iteration when dealing with contractive like operators", Fixed Point Theory and Applications, 2008(2008), Article ID 242916, 7 pages.

[22] T. Zamfirescu,"Fixed point theorems in metric spaces", Archiv der Mathematik, 23(1972), no.1, 292-298.

[23] V. Berinde,"Iterative Approximation of Fixed Points ", Springer, Berlin (2007).

[24] V. Berinde,"On the approximation of fixed points of weak contractive mappings", Carpathian J. Math., 19(2003), no. 1, 7-22.
[25] W. Phuengrattana, S. Suantai,"On the rate of convergence of Mann, Ishikawa, Noor and SP iterations for continuous functions on an arbitrary interval", Journal of Computational and Applied Mathematics, 235(2011), 3006-3014.

[26] W. Takahashi,"Iterative methods for approximation of fixed points and their applications", Journal of the Operations Research Society of Japan, 43(2000), no.1, 87-108.

[27] W.R. Mann, "Mean value methods in iterations", Proc. Amer. Math. Soc., 4(1953), 506-510.

[28] Xu, M.A. Noor, "Ishikawa and Mann iteration process with errors for nonlinear strongly accretive operator equations", J. Math. Anal. Appl., 224(1998), 91-101 